\title{
Rapid progression of a granulocyte colony-stimulating factor-producing liver tumor metastasized from esophagogastric junction cancer: A case report and literature review
}

\author{
SOJUN HOSHIMOTO $^{1}$, NOBUO HOSHI ${ }^{2}$, IWAO OZAWA ${ }^{1}$, MORIAKI TOMIKAWA ${ }^{1}$, \\ HIROFUMI SHIRAKAWA ${ }^{1}$, TAKESHI FUJITA ${ }^{1}$, SAHO WAKAMATSU ${ }^{2}$, SAYURI HOSHI $^{2}$, \\ KAORU HIRABAYASHI ${ }^{2}$, SHOICHI HISHINUMA ${ }^{1}$ and YOSHIRO OGATA ${ }^{1}$ \\ Departments of ${ }^{1}$ Digestive Surgery and ${ }^{2}$ Pathology, Tochigi Cancer Center, Utsunomiya, Tochigi 320-0834, Japan
}

Received October 21, 2017; Accepted February 22, 2018

DOI: $10.3892 / \mathrm{ol} .2018 .8144$

\begin{abstract}
The current study presents the case of a 72-year-old woman with a rapidly enlarged liver metastasis from esophagogastric junction (EGJ) cancer, accompanied by progressive leukocytosis $(47,680 / \mu 1)$ and elevated serum granulocyte colony-stimulating factor (G-CSF; $779 \mathrm{pg} / \mathrm{ml})$. The patient underwent right hemihepatectomy 26 months after a total gastrectomy. On the seventh post-operative day the patient's leukocyte count and serum G-CSF level decreased to 4,280/ $\mu \mathrm{l}$ and $\leq 19.5 \mathrm{pg} / \mathrm{ml}$, respectively. Histologically, the lesion was a well to moderately differentiated adenocarcinoma similar to the primary lesion. Therefore, this tumor was clinically diagnosed as a G-CSF-producing liver metastasis from EGJ cancer, although immunohistochemical staining for G-CSF was negative. A right pulmonary nodule detected simultaneously with the hepatic mass was resected four months following the hepatectomy and was diagnosed as a pulmonary metastasis. The patient's leukocyte count was normal at the time of her initial surgery for EGJ cancer, and her clinical course varied for different metastatic sites. The liver metastasis was accompanied by progressive leukocytosis and elevated serum G-CSF and demonstrated rapid tumor growth during a six-month period, whereas the non-G-CSF-producing pulmonary metastasis grew slowly during the same period. In addition 21 reported cases of G-CSF-producing upper gastrointestinal tract cancer were reviewed to elucidate the clinicopathological features of this disease.
\end{abstract}

Correspondence to: Dr Sojun Hoshimoto, Department of Digestive Surgery, Tochigi Cancer Center, 4-9-13, Yohnan, Utsunomiya, Tochigi 320-0834, Japan

E-mail: souhoshi@tochigi-cc.jp

Key words: esophagogastric junction cancer, granulocyte colony-stimulating factor, leukocytosis, upper gastrointestinal tract cancer

\section{Introduction}

Tumor-related leukocytosis, which is occasionally encountered in patients with malignant tumors, is a paraneoplastic syndrome involving high leukocyte counts without underlying infection, bone marrow metastasis, or corticosteroid administration $(1,2)$. Tumor-related leukocytosis is observed in $10 \%$ of patients with solid tumors and is associated with poor clinical outcome (3). This condition is caused, in part, by granulocyte colony-stimulating factor (G-CSF) synthesized by neoplastic cells and has therefore been regarded as potentially indicative of the autocrine stimulation of tumor growth by G-CSF $(4,5)$. However, little is known regarding the precise mechanisms of aggressive behavior and poor outcome in G-CSF-producing tumors. G-CSF production by tumors has been reported for various non-hematopoietic malignancies, predominantly lung or pancreatic cancer $(6,7)$. On the other hand, G-CSF-producing cancer originated from upper gastrointestinal tract including esophagus, esophagogastric junction (EGJ), stomach is extremely rare, and the clinical characteristics and outcomes of this entity remain unclear.

Here, we present a patient with a rapidly enlarged liver metastasis from EGJ cancer accompanied by progressive leukocytosis and high levels of serum G-CSF, although her leukocyte count had been normal two years earlier at the first surgery for the primary lesion. Additionally, we review 21 cases of G-CSF-producing upper gastrointestinal tract cancer, including 20 previously published cases and the present case, to elucidate the clinicopathological features of this entity.

\section{Case report}

A 72-year-old woman had undergone laparoscopic total gastrectomy with regional lymph node dissection for EJG cancer [pT3N1M0, stage IIB according to the Union for International Cancer Control (UICC) TNM classification (8)]. Histopathological examination revealed well to moderately differentiated adenocarcinoma with a $3+$ score for human epidermal growth factor receptor 2 (HER2) expression (9). Atrophic gastritis positive for $H$. pylori infection was identified in the pyloric gland area. The patient received adjuvant 
chemotherapy with S-1 for a year and did not experience tumor recurrence or metastasis prior to the events described here. Before gastrectomy, her leukocyte count was 7,770/ $\mu 1$, with $68.8 \%$ neutrophils. During follow-up, the leukocyte count increased to $27,150 / \mu 1$ with $87.0 \%$ neutrophils at the 21 st month after gastrectomy, but the serum C-reactive protein concentration was within a normal range $(0.07 \mathrm{mg} / \mathrm{dl})$. Bone marrow aspiration biopsy revealed a hypercellular marrow with predominantly granulocytic mature cells. Molecular analyses revealed neither a BCR/ABL fusion gene nor the JAK2-V617F mutation. The patient's serum G-CSF level was significantly elevated (779 pg/ml; normal, $<39$ pg/ml). Contrast-enhanced computed tomography (CT) during the 24th postoperative month revealed a low-density mass measuring approximately $80 \mathrm{~mm}$ in diameter in the right lobe of the liver, with irregular and peripheral enhancement of the lesion in the arterial phase and wash-out in the portal and delayed phases (Fig. 1). Furthermore, a small nodule with a size of $6 \mathrm{~mm}$ was identified in the right lower lobe of the lung. The hepatic mass had not been detected on abdominal CT performed 6 months previously, suggesting rapid tumor growth. Although the patient did not exhibit symptoms, her leukocyte count continued to increase, reaching 47,680/ $\mu \mathrm{l}$ during the 26th postoperative month. As other potential causes of leukocytosis were excluded, we hypothesized that this condition was a paraneoplastic manifestation induced by G-CSF production by the liver tumor. Based on appropriate radiological findings for hepatocellular carcinoma, the lesion was diagnosed as G-CSF-producing hepatocellular carcinoma, with liver metastasis from EGJ cancer included as a differential diagnosis, and the patient underwent right hemihepatectomy 26 months after gastrectomy. The resected specimen included a hard, solid, whitish tumor measuring 99x79x63 mm in size in the right lobe of the liver (Fig. 2A). Histopathological examination revealed a well to moderately differentiated adenocarcinoma (Fig. 2B). The tumor in the liver and the primary lesion had similar histology and identical immunohistochemical (IHC) patterns, including reactivity to cytokeratin (CK)7, CK19, CK20, and carcinoembryonic antigen (CEA). The HER2 expression score (9) of the liver tumor was 3+, as was that of the primary lesion (Fig. 2C). IHC examination using an anti-G-CSF monoclonal antibody revealed negative G-CSF expression (Fig. 2D). However, the patient's leukocyte count and serum G-CSF level had dramatically decreased to $4,280 / \mu 1$ and $\leq 19.5 \mathrm{pg} / \mathrm{ml}$, respectively, on the seventh postoperative day. Therefore, clinically, the tumor was diagnosed as G-CSF-producing liver metastasis from EGJ cancer, although IHC staining for G-CSF was negative. The right pulmonary mass had slightly expanded to $8 \mathrm{~mm}$ on chest CT performed during the 28th postoperative month (Fig. 1B). However, the patient's leukocyte count $(3,650 / \mu 1)$ and serum G-CSF level $(32.0 \mathrm{pg} / \mathrm{ml})$ were in the normal range. Thoracoscopic partial pulmonary resection was performed four months after hepatectomy. Histological examination of the pulmonary mass revealed similar findings to those obtained for the primary lesion and the liver metastasis, and the HER2 expression score (9) of the pulmonary tumor was $3+$. Thus, this mass was diagnosed as pulmonary metastasis. We decided not to proceed with adjuvant chemotherapy after pulmonary resection because no other obvious recurrence was identified. The patient's leukocyte count and serum G-CSF have continued to remain at normal levels during follow-up (Fig. 3). She has experienced no tumor recurrence and has survived 38 and 12 months after gastrectomy and hepatectomy, respectively.

\section{Discussion}

In general, the following criteria are used as the diagnostic standard for detecting a G-CSF-producing tumor: (1) an increased number of leukocytes, primarily mature neutrophils, with no other explanatory factors; (2) elevated serum G-CSF; (3) a reduction in the leukocyte count following tumor resection; and (4) confirmation of G-CSF production via immunostaining (10). However, clinically, positive immunostaining is not a definitive finding for the diagnosis of G-CSF-producing tumors (11). It has been suggested that the rapid secretion of G-CSF without intracellular retention is the reason why such tumors may exhibit negative staining with an anti-G-CSF monoclonal antibody (12). In fact, Yokoyama et al (13), reported a case involving G-CSF-producing gastric cancer that exhibited negative IHC staining for G-CSF but highly increased G-CSF mRNA levels detected using real-time reverse transcription polymerase chain reaction. In the present case, postoperative observations indicated that criteria (1) to (3) were fulfilled, and the lesion satisfied the criteria for a G-CSF-producing tumor.

In the present case, a normal leukocyte count was initially detected at the first surgery for the primary EGJ cancer, and the clinical course varied for different metastatic sites. Interestingly, the liver metastasis, which was accompanied by progressive leukocytosis and elevated serum G-CSF, showed rapid tumor growth during a six-month period, whereas the subsequent pulmonary metastasis, which was associated with a normal leukocyte count and normal serum G-CSF, grew slowly during the same time period. These findings indicate the existence of tumor cell heterogeneity in different metastatic sites or a subset of cancer cells acquiring the ability to produce G-CSF that could respond to G-CSF with enhanced proliferation, a phenomenon suggestive of autocrine mechanisms and of more aggressive biological activities for such cells compared with that of cancer cells that do not produce G-CSF.

In a PubMed search, 14 case reports (13-26) and 6 cited publications $(11,27-31)$ regarding G-CSF-producing upper gastrointestinal tract cancers, including esophageal cancer (12 cases), EGJ cancer (1 case), and gastric cancer ( 7 cases), were retrieved from the English-language literature. Demographic and clinical characteristics for all of the described cases of such cancers, including the present case, are summarized in Table I. A male predominance was identified in these 21 cases (90\% vs. 10\%). Interestingly, male predilection was also observed in G-CSF-producing pancreatic cancer (85\% vs. $15 \%$ ) (7). However, the association of sex predominance with G-CSF production in cancer tissue is unclear. Pyrexia was observed in 7 of these 21 cases, although the patient described here remained asymptomatic despite her large tumor burden. Two cases and one case of G-CSF-producing gastric cancer were histologically diagnosed as adenosquamous carcinoma and undifferentiated carcinoma, respectively. Among the 21 described cases, neutrophil infiltration in the tumor was observed in only two cases of G-CSF-producing esophageal 

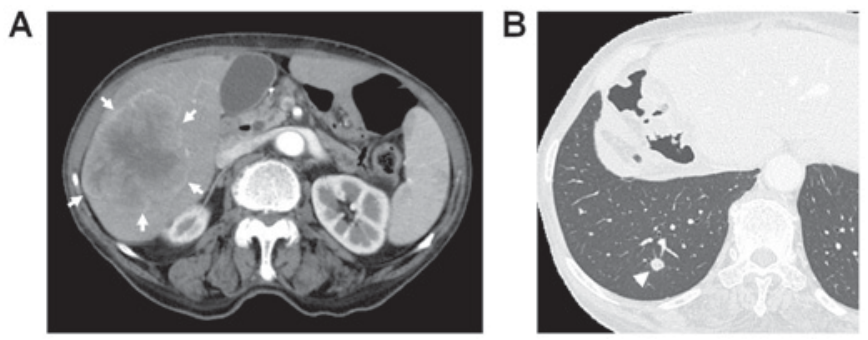

Figure 1. Imaging studies. (A) Abdominal CT revealed a low-density tumor measuring $\sim 80 \mathrm{~mm}$ in diameter in the right lobe of the liver with irregular and peripheral enhancement of the lesion in the arterial phase and wash-out in the portal and delayed phases (white arrows). (B) A Chest CT revealed a right pulmonary tumor that slowly increased from 6 to $8 \mathrm{~mm}$ during a six-month period (white arrow head). CT, computed tomography.
A

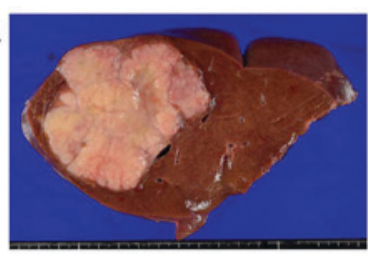

c

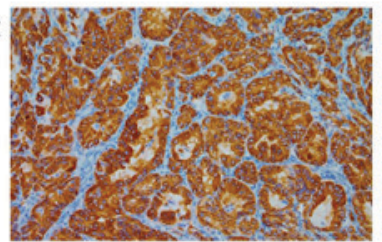

B

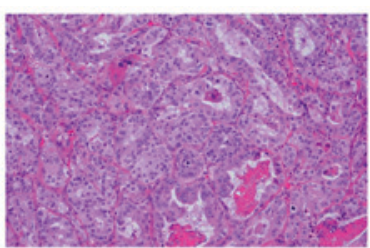

D

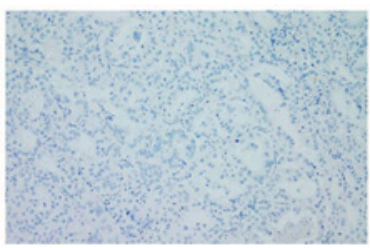

Figure 2. Pathology of the liver tumor. (A) Macroscopic appearance of the resected specimen. The tumor was a well-defined, whitish mass lesion with a diameter of $99 \mathrm{~mm}$. (B) Microscopic evaluation following hematoxylin-eosin staining of the tumor tissue revealed a well- to moderately differentiated adenocarcinoma (magnification, x200). (C) IHC examination revealed human epidermal growth factor receptor 2 expression scores of $3+$ for the liver tumor (magnification, x200). (D) IHC examination revealed negative granulocyte colony-stimulating factor expression (magnification, x200). IHC, immunohistochemical.

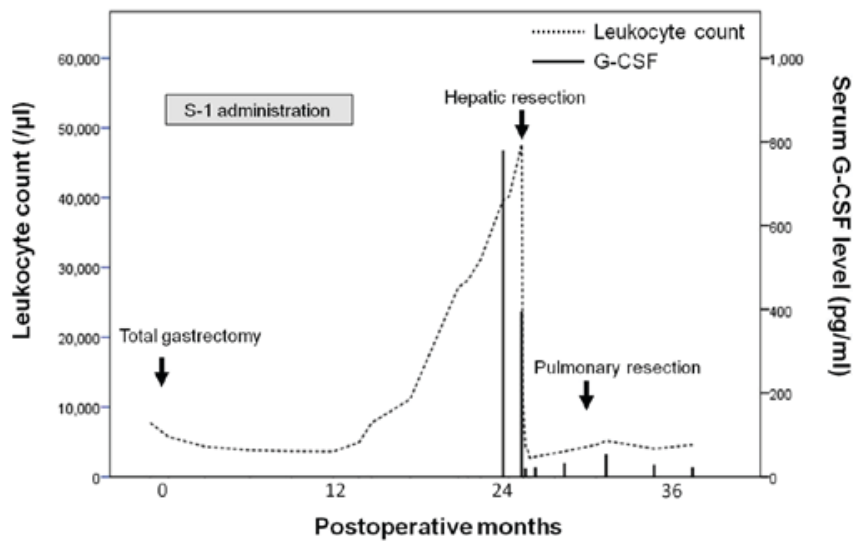

Figure 3. Clinical course and changes in leukocyte count and the level of serum G-CSF. G-CSF, granulocyte colony-stimulating factor.

cancer $(16,25)$. However, no specific morphological features were found. In addition, this is the first reported case of G-CSF-producing upper gastrointestinal tract cancer with HER2 expression. However, to the best of our knowledge, no studies have demonstrated correlations or interactions between HER 2 and G-CSF. Among the 21 described cases, 14 cases involved surgical resection, whereas the remaining 7 cases involved chemo- and/or radiation therapy or the best supportive care due to advanced disease or deterioration of the patient's general condition. Overall survival in these 21 cases ranged from 2 to 24 months. For the 13 cases involving resection, excluding a case of gastric cancer that exhibited G-CSF production only after recurrence, resection of the G-CSF-producing tumor was followed by a decrease in leukocyte count, with normal leukocyte levels reached in 10 of these 13 cases. However, in 8 of 13 cases, an increase in leukocyte count and recurrence were observed during the early postoperative period, and 6 patients died of their disease within 2 years after surgical resection. In addition, chemotherapy and/or radiotherapy decreased the leukocyte counts and serum G-CSF levels in accordance with the remission of tumors in five cases $(15,21,28,30,31)$. These findings suggest that leukocyte count or serum G-CSF level would be useful for monitoring disease in cases involving G-CSF-producing tumors, even if conventional biomarkers are negative. The patient described here is the first reported case of GCSF-producing upper gastrointestinal tract cancer that underwent surgical resection of distant organ metastasis. The patient has survived for 38 months and 12 months after surgical resection of the non-G-CSF-producing primary lesion and the G-CSF-producing liver metastasis, respectively. Little is known about surgical indications for patients with EGJ cancer who undergo surgery for the primary lesion and experience recurrence. Depypere et al (32), analyzed 1754 patients surgically treated with curative resection for esophageal cancer and EGJ cancer and reported a 49.9\% 5-year overall survival rate in patients who underwent surgical resection of isolated local recurrence or solitary solid organ metastasis with or without systemic therapy. However, a multidisciplinary team is needed for the optimal management of the recurrence of EGJ cancer, and surgery should be limited to selected patients, especially when considering the surgical indication for patients with G-CSF-producing EGJ cancer given its rapid progression and unfavorable prognosis. In the present case, hepatectomy was planned because the liver tumor was initially diagnosed as G-CSF-producing hepatocellular carcinoma based on radiological findings. In addition, pulmonary resection was performed because the lung tumor grew slowly without leukocytosis, unlike the liver tumor, and primary lung cancer was included as a differential diagnosis.

There have been a few reported cases of G-CSF-producing gastric cancer similar to the present case in that metastatic disease or local recurrence was present when paraneoplastic leukocytosis was detected. Kawaguchi et al (17), reported a case of gastric cancer with an aggressive course after recurrence in the liver and lymph nodes as a G-CSF-producing tumor, despite the fact that the primary lesion did not exhibit G-CSF production; the patient in question died of the disease only two months after surgery. Moreover, Yamano et al (26), reported a case of G-SCF-producing gastric cancer in which rapid progression of the residual tumor was observed after endoscopic mucosal resection. In that case, histological analysis of the tumor resected via endoscopic mucosal resection revealed a well-differentiated adenocarcinoma with negative immunoreactivity for G-CSF, whereas the residual 


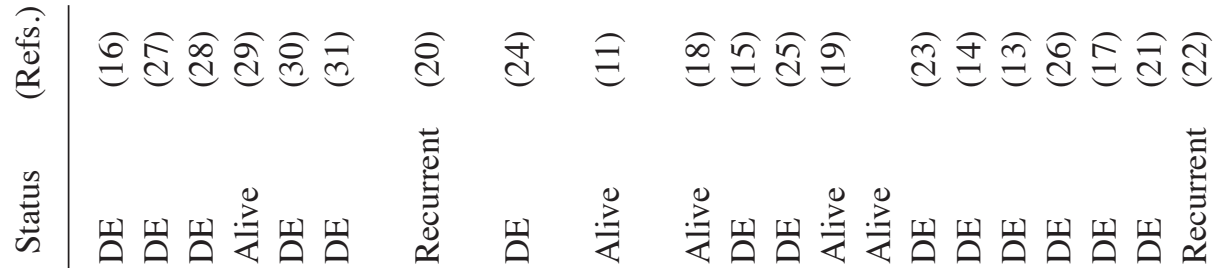

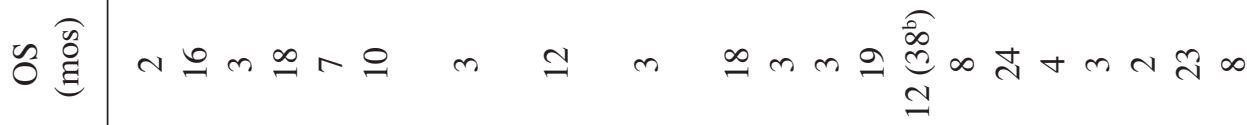

$\stackrel{0}{>}$

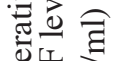

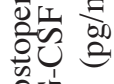
ㄴำ

$\stackrel{\overbrace{}}{\Xi}$

产苍苍

过

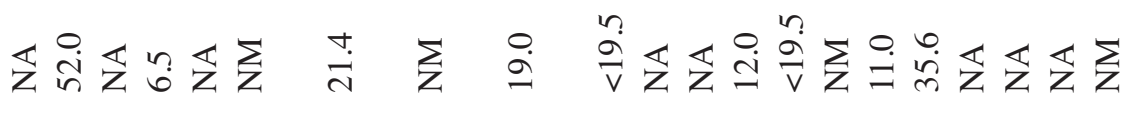

.

iิ

.

品

焉.

离

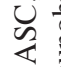

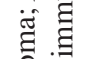

言品

这

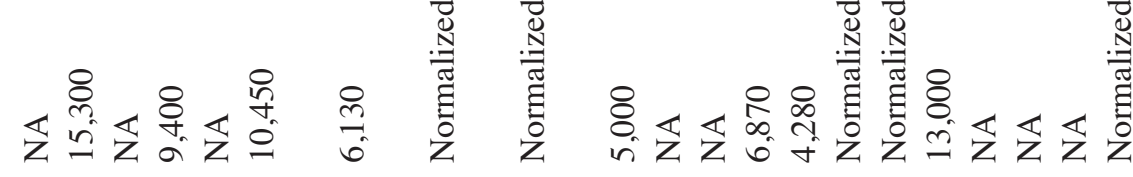

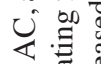

它要 穵

离

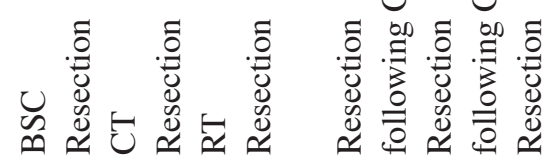

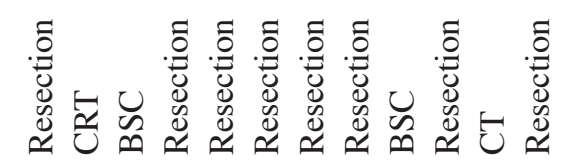

ए.

焉焉

苛

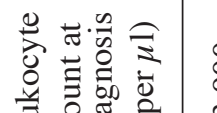

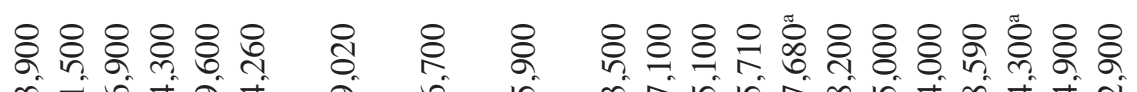

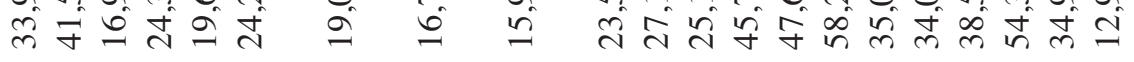

竞剀

焉证

总

总|

竞

总

要

蔍

嵒市

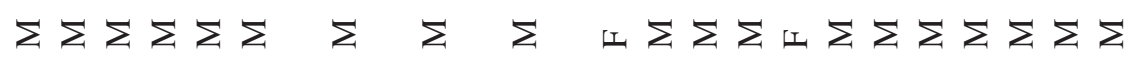

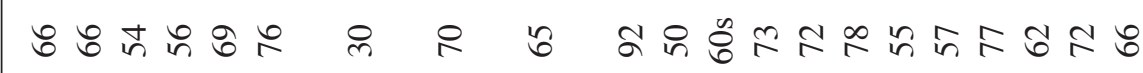

营

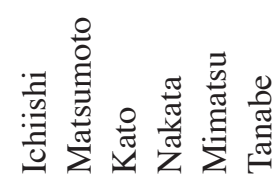

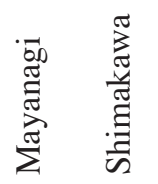

$\frac{7}{3}$

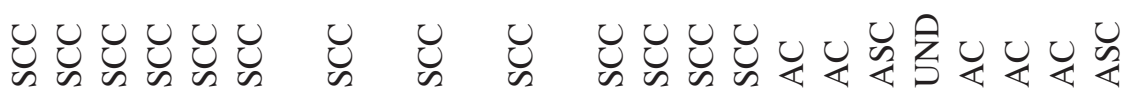

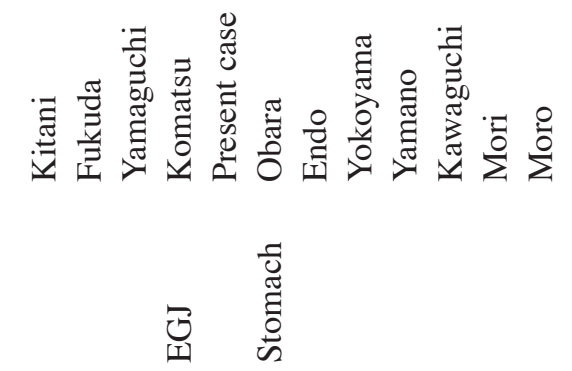

의 홋 뗘

要

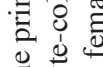

동

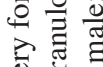

然

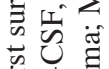

密

要

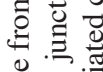

色

语。

密

o :

焉完

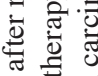

글흘 항

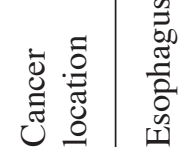

总离

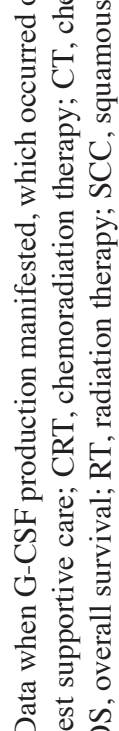


tumor, which showed rapid tumor growth, presented as a poorly differentiated adenocarcinoma with positive immunoreactivity for G-CSF; this finding suggested that histological change in the tumor may have influenced G-CSF production and induced rapid progression. However, in the present case, histological evaluation of the liver metastasis revealed a well to moderately differentiated adenocarcinoma similar to the primary lesion, and there was no evidence of dedifferentiation or anaplastic transformation. In addition, we assessed the tumor proliferative ability of each lesion by immunostaining for Ki-67, as previously described (33). Notably, the Ki-67 index of the liver metastasis $(58.4 \%)$ was similar to the $\mathrm{Ki}-67$ indices of the primary lesion and the pulmonary metastasis (65.0 and $73.6 \%$, respectively). In addition, the presence of tumor-infiltrating lymphocytes in liver and lung metastasis was similar, whereas abundant stromal myofibroblasts with elevated expression of $\alpha$-smooth muscle actin was observed in only liver metastasis. No differences in CD3+ lymphocyte and CD20+ lymphocyte counts were noted between normal liver and lung tissue surrounding the tumor. These findings suggest that morphological and biological characteristics remained unchanged throughout the clinical course of the described case, even though rapid tumor growth and elevated G-CSF were only observed during the course of the liver metastasis.

$\mathrm{G}-\mathrm{CSF}$ has been reported to promote tumor progression in different tumor models. A prior study demonstrated that G-CSF could promote the survival and activation of myeloid-derived suppressor cells via the signal transducer and activator of transcription 3 (STAT3) signaling pathway, resulting in the induction of immune suppression (34). Furthermore, G-CSF is known to regulate epithelial to mesenchymal transition (EMT) via recruitment of the $c$-jun proto-oncogene. Moreover, in previous studies, G-CSF increased the proliferation and migration of tumor cells in a manner dependent on ERK1/2 and RSK1 phosphorylation (4) and stimulated tumor angiogenesis $(35,36)$. However, the precise mechanisms of G-CSF-dependent autocrine growth and proliferation are not well known.

Clinically, a more aggressive course is sometimes observed in the metastatic site than in the primary lesion. The case described here suggests that one of the mechanisms of this biological change might be due to tumor cell heterogeneity in different metastatic sites or to a subset of cancer cells acquiring the ability to produce G-CSF. The present case involved successful treatment with radical surgery for a G-CSF-producing liver metastasis and was distinct from previously described cases in that the patient exhibited long-term survival. Given the unfavorable prognosis and relatively high probability of treatment failure associated with G-CSF-producing tumors, optimal diagnostic and therapeutic approaches and careful monitoring for the early detection of recurrence should be considered for patients with such tumors.

\section{Acknowledgements}

Not applicable.

\section{Funding}

No funding was received.

\section{Availability of data and materials}

Data sharing is not applicable to this article as no datasets were generated or analyzed during the current study.

\section{Authors' contributions}

SoH and NH designed the report. MT, TF, HS, IO, ShH and YO treated the patient and contributed to the collection of the clinical data. SoH analyzed the data and wrote the manuscript. NH, ShH and IO reviewed and edited the manuscript. $\mathrm{NH}, \mathrm{SW}, \mathrm{SaH}$ and $\mathrm{KH}$ reviewed the pathological findings, performed immunohistochemical staining and prepared the pathological images.

\section{Ethics approval and consent to participate}

Not applicable.

\section{Consent for publication}

The patients provided written informed consent for the publication of their clinical data and images.

\section{Competing interests}

The authors declare that they have no competing interests.

\section{References}

1. Bahar B, Acedil Ayc Iota B, Çoşkun U, Büyükberber S, Benekli M and Yildiz R: Granulocyte colony stimulating factor (G-CSF) and macrophage colony stimulating factor (M-CSF) as potential tumor markers in non small cell lung cancer diagnosis. Asian Pac J Cancer Prev 11: 709-712, 2010.

2. Kawano M, Mabuchi S, Matsumoto Y, Sasano T, Takahashi R, Kuroda H, Kozasa K, Hashimoto K, Isobe A, Sawada K, et al: The significance of G-CSF expression and myeloid-derived suppressor cells in the chemoresistance of uterine cervical cancer. Sci Rep 5: 18217, 2015.

3. Granger JM and Kontoyiannis DP: Etiology and outcome of extreme leukocytosis in 758 nonhematologic cancer patients: A retrospective, single-institution study. Cancer 115: 3919-3923, 2009.

4. Morris KT, Khan H, Ahmad A, Weston LL, Nofchissey RA, Pinchuk IV and Beswick EJ: G-CSF and G-CSFR are highly expressed in human gastric and colon cancers and promote carcinoma cell proliferation and migration. Br J Cancer 110: 1211-1220, 2014

5. Shimamura K, Fujimoto J, Hata J, Akatsuka A, Ueyama Y, Watanabe T and Tamaoki N: Establishment of specific monoclonal antibodies against recombinant human granulocyte colony-stimulating factor (hG-CSF) and their application for immunoperoxidase staining of paraffin-embedded sections. J Histochem Cytochem 38: 283-286, 1990.

6. Aliper AM, Frieden-Korovkina VP, Buzdin A, Roumiantsev SA and Zhavoronkov A: A role for G-CSF and GM-CSF in nonmyeloid cancers. Cancer Med 3: 737-746, 2014.

7. Vinzens S, Zindel J, Zweifel M, Rau T, Gloor B and Wochner A: Granulocyte colony-stimulating factor producing anaplastic carcinoma of the pancreas: Case report and review of the literature. Anticancer Res 37: 223-228, 2017.

8. Sobin LH, Gospodarowicz MK and Wittekind Ch: TNM classification of malignant tumours. Wiley-Blackwell, Hoboken, NJ, 2010.

9. Wolff AC, Hammond ME, Hicks DG, Dowsett M, McShane LM, Allison KH, Allred DC, Bartlett JM, Bilous M, Fitzgibbons P, et al: Recommendations for human epidermal growth factor receptor 2 testing in breast cancer: American Society of Clinical Oncology/College of American Pathologists clinical practice guideline update. J Clin Oncol 31: 3997-4013, 2013. 
10. Hanaoka T, Jingu K, Tochigi T, Hoshino I, Uematu T and Matsubara H: A case of G-CSF-producing histiocytic sarcoma of the stomach. Int Surg 100: 568-573, 2015.

11. Oshikiri T, Yasuda T, Harada H, Ohyama M, Hasegawa H, Ohara T, Sendo H, Sugimoto T, Fujino Y, Tominaga M and Takahash Y: G-CSF-producing esophageal cancer with induction of intense bone marrow FDG uptake. Esophagus 12: pp258-262, 2015.

12. Katoh Y, Nakamura M, Ohnishi Y, Shimamura K, Ueyama Y and Tamaoki N: Autonomous production of granulocyte-colony stimulating factor in tumour xenografts associated with leukocytosis. Br J Cancer 68: 715-719, 1993.

13. Yokoyama T, Hyodo M, Hosoya Y, Koinuma K, Kurashina K, Saitoh S, Hirashima Y, Arai W, Zuiki T, Yasuda Y, et al: Aggressive G-CSF-producing gastric cancer complicated by lung and brain abscesses, mimicking metastases. Gastric Cancer 8: 198-201, 2005

14. Endo K, Kohnoe S, Okamura T, Haraguchi M, Adachi E, Toh Y, Baba H and Maehara Y: Gastric adenosquamous carcinoma producing granulocyte-colony stimulating factor. Gastric Cancer 8: 173-177, 2005.

15. Fukuda S, Fujiwara Y, Mishima H, Wakasa T, Hanamoto $\mathrm{H}$ Inoue $\mathrm{K}$, Kitani $\mathrm{K}$, Ishikawa $\mathrm{H}$, Tsujie $\mathrm{M}$, Yukawa M, et al: Choroidal metastasis from granulocyte colony-stimulating factor-producing esophageal squamous cell carcinoma: A case report. Clin Case Rep 5: 419-424, 2017.

16. Ichiishi E, Yoshikawa T, Kogawa T, Yoshida N and Kondo M: Possible paracrine growth of adenocarcinoma of the stomach induced by granulocyte colony stimulating factor produced by squamous cell carcinoma of the oesophagus. Gut 46: 432-434, 2000.

17. Kawaguchi M, Asada Y, Terada T, Takehara A, Munemoto Y, Fujisawa K, Mitsui T, Iida Y, Miura S and Sudo Y: Aggressive recurrence of gastric cancer as a granulocyte-colony-stimulating factor-producing tumor. Int J Clin Oncol 15: 191-195, 2010.

18. Kitani M, Yamagata Y, Tanabe A, Yagi K, Aikou S, Kiyokawa T, Nishida M, Yamashita H, Mori K, Nomura S and Seto Y: Radical esophagectomy for a 92-year-old woman with granulocyte colony-stimulating factor-producing esophageal squamous cell carcinoma: A case report. World J Surg Oncol 14: 264, 2016.

19. Komatsu D, Sakurai M, Nakafuji H, Koide N, Morishita H and Nakamura T: Granulocyte colony stimulating factor-producing collision tumor of the gastric cardia. J Gastroenterol 38 1013-1015, 2003.

20. Mayanagi S, Niihara M, Goto H, Yokota T, Tabuse H, Yasui H, Ogawa H, Nishimura T, Kusafuka K and Tsubosa Y: Granulocyte colony-stimulating factor-producing esophageal squamous cell carcinoma following chemoradiotherapy and bone marrow transplantation for acute lymphoblastic leukemia. Esophagus 10 258-263, 2013

21. Mori H, Shibuya T, Osada T, Kodani T, Higashihara Y, Serizawa N, Kato J, Nagahara A, Ogihara T and Watanabe S: Response to chemotherapy in a case of gastric adenocarcinoma producing granulocyte colony-stimulating factor. Med Sci Monit 16: CS119-CS123, 2010

22. Moro K, Nagahashi M, Naito T, Nagai Y, Katada T, Minagawa M, Hasegawa J, Tani T, Shimakage N, Usuda H, et al: Gastric adenosquamous carcinoma producing granulocyte-colony stimulating factor: A case of a rare malignancy. Surg Case Rep 3: 67, 2017.

23. Obara T, Ito Y, Kodama T, Fujimoto Y, Mizoguchi H, Oshimi K, Takahashi M and Hirayama A: A case of gastric carcinoma associated with excessive granulocytosis. Production of a colony-stimulating factor by the tumor. Cancer 56: 782-788, 1985.
24. Shimakawa T, Asaka S, Usuda A, Yamaguchi K, Yoshimatsu K, Shiozawa S, Katsube T and Naritaka Y: Granulocyte-colony stimulating factor (G-CSF)-producing esophageal squamous cell carcinoma: A case report. Int Surg 99: 280-285, 2014.

25. Yamaguchi S, Kanetaka K, Kobayashi S, Nagata Y, Kinosita N, Fukuoka J, Murakami S, Fujita F, Takatsuki M and Eguchi S: Severe neutrophilic leukocytosis as a progression marker in granulocyte colony-stimulating factor-producing squamous cell carcinoma of the esophagus. Clin Case Rep 5: 688-693, 2017.

26. Yamano T, Morii E, Ikeda J and Aozasa K: Granulocyte colony-stimulating factor production and rapid progression of gastric cancer after histological change in the tumor. Jpn J Clin Oncol 37: 793-796, 2007.

27. Matsumoto G, Ise H, Kimura Y, Inoue H, Suzuki N, Ohtani H, Ogawa H, Fukushima K and Matsuno S: Granulocyte-colony stimulating factor-producing esophageal carcinoma: Serum level as a marker for monitoring the effects of treatment. Int J Clin Oncol 5: pp328-333, 2000.

28. Kato M, Osawa H, Usui $\mathrm{N}$ and Hano $\mathrm{H}$ : An autopsy case of esophageal squamous cell carcinoma associated with granulocyte colony-stimulating factor production (case report). Jikeikai Med J 49: 191-195, 2002.

29. Nakata K, Ohtsuka T, Sato S, Tanaka M, Shimonishi T, Mori D, Nakafusa Y and Miyazaki K: Esophageal carcinoma with humoral hypercalcemia and leukocytosis successfully treated by a two-stage operation: Report of a case. Esophagus 3: pp13-17, 2006.

30. Mimatsu K, Oida T, Kano H, Kawasaki A and Amano S: Aggressive progression of granulocyte colony-stimulating factor producing squamous cell carcinoma of the esophagus: Case report and literature review. Esophagus 5: pp205-209, 2008.

31. Tanabe T, Kanda T, Ishihara N, Kosugi S-I, Matsuki A, Watanabe G, Sasamoto R and Hatakeyama K: An esophageal squamous cell carcinoma patient with high serum granulocyte-colony stimulating factor level: Report of a case. Esophagus 6: 253, 2009 (https://doi.org/10.1007/s10388-009-0206-z).

32. Depypere L, Lerut T, Moons J, Coosemans W, Decker G, Van Veer H, De Leyn P and Nafteux P: Isolated local recurrence or solitary solid organ metastasis after esophagectomy for cancer is not the end of the road. Dis Esophagus 30: 1-8, 2017.

33. Hoshimoto S, Hoshi S, Hishinuma S, Tomikawa M, Shirakawa $H$, Ozawa I, Wakamatsu S, Hoshi N, Hirabayashi K and Ogata Y: Adenosquamous carcinoma in the biliary tract: Association of the proliferative ability of the squamous component with its proportion and tumor progression. Scand J Gastroenterol 52: 425-430, 2017

34. Li W, Zhang X, Chen Y, Xie Y, Liu J, Feng Q, Wang Y, Yuan W and Ma J: G-CSF is a key modulator of MDSC and could be a potential therapeutic target in colitis-associated colorectal cancers. Protein Cell 7: 130-140, 2016.

35. Natori T, Sata M, Washida M, Hirata Y,Nagai R and Makuuchi M: G-CSF stimulates angiogenesis and promotes tumor growth: Potential contribution of bone marrow-derived endothelial progenitor cells. Biochem Biophys Res Commun 297: 1058-1061, 2002.

36. Voloshin T, Gingis-Velitski S, Bril R, Benayoun L, Munster M, Milsom C, Man S, Kerbel RS and Shaked Y: G-CSF supplementation with chemotherapy can promote revascularization and subsequent tumor regrowth: Prevention by a CXCR4 antagonist. Blood 118: 3426-3435, 2011. 